\title{
Gastroesophageal Junction Adenocarcinoma by AJCC v8 Stage
}

National Cancer Institute

\section{Source}

National Cancer Institute. Gastroesophageal Junction Adenocarcinoma by A/CC v8 Stage. NCl Thesaurus. Code C133548.

A term that refers to the staging of gastroesophageal junction adenocarcinoma according to the American Joint Committee on Cancer, 8th edition. 